\title{
Design and Fabrication of Low Speed Wind Tunnel
}

\author{
Akshay Patil \\ B Tech. \\ MIT-World Peace University \\ Pune, India \\ akshay8066@gmail.com
}

\author{
Kaustubh Kulkarni \\ Assistance Professor \\ MIT-World Peace University \\ Pune, India
}

\begin{abstract}
A slow wind tunnel should be designed and built. This project involved the design and construction of the small wind tunnel. During this project, a computer aided design (CAD) called ANSYS will be used to design the wind tunnel.
\end{abstract}

Objectives: This report explains the entire design process of an open-circuit subsonic wind tunnel that studies the effect of wind on different prototypes of structural elements. Method: Existing guidelines and previous research results were followed in calculating the different parts of the wind tunnel. Construction tests were carried out to reach a speed of $20 \mathrm{~m} /$ $\mathrm{s}$ in the test section. The latest attempt was discussed in the main body of the report.

Findings: The construction includes a square test section with a side length of $200 \mathrm{~mm}$ to accommodate the model and tools needed for force and pressure measurement. A straight section is provided in front of the test chamber to allow the outlet of the contraction section to stabilize before it reaches the test section. Instead of a curved wall shape, a straight contraction profile with a longer contraction length is used. Application: The ventilator was used to visualize the separation of flows of different bodies contained in the test section using smoke.

\section{INTRODUCTION}

In aerodynamic research, a wind tunnel is used to study the effects of air passing in front of solid objects. A wind tunnel consists of a tubular passage, in the center of which the object to be tested is mounted. Air is pushed past the object by a powerful ventilation system or other means. As a test object, we measure aerodynamic forces, pressure distribution or other aerodynamic properties. The first wind tunnels were invented in the late 19th century, at the beginning of aeronautical research, when many attempted to create successful flying machines heavier than air. Wind tunnel tests were applied to automobiles, not so much to determine the aerodynamic forces per se, but rather to reduce the power required to move the vehicle on roads at a given speed. The interaction between road and vehicle plays an important role in these studies and this interaction should be considered when interpreting the test results. In a real situation, the pavement moves relative to the vehicle, but the air is still relative to the roadway, but in the wind tunnel, the air moves relative to the roadway while the roadway is stationary relative to the test vehicle. Some Auto test wind tunnels have movable belts installed under the test vehicle to approximate actual conditions, and very similar devices are used in wind tunnel tests of aircraft take-off and landing configurations. [1]

- A wind tunnel is used to simulate the flow of air on an airplane model or section of a wing in order to study it.

- The model can be equipped with tools to measure lift and drag, as well as other parameters of interest.

- The fan can create a flow of air at the desired speed and state.

- Engineers can use a wind tunnel to study and design the shape of a wing.

- Wind tunnels can be used to study interference between aircraft parts or between an aircraft and a bomb when it falls.

\section{LITERATURE REVIEW}

Sahith Reddy Madara et al. [3] This work predominantly centres around incorporating the association of a little subsonic wind current, analysing the stream portrayal on an article, and assessing the lift and drag coefficient of an article by testing. As of now, when measurements are reached with immense stores of consumption, practically all airplane are gathered utilizing CAD programming. Persuasive examination incorporates not just affirming the state of the withdrawing cone considering the air speed in various areas, which were tackled in a CFD concentrate with exploratory properties in relating powers.

Amol L. Mangrulkar et al. [4] This article incorporates a discussion about arranging and gathering an unassuming wind current that can be utilized for instructive purposes. The objective was to make satisfactory wind stream at a speed of over $10 \mathrm{~m} / \mathrm{s}$ in the test fragment and to incorporate factors like traditionalism, solace and robustness being developed. Another objective was to direct an investigation on comparable proliferation and show the differentiation among numerical and exploratory outcomes.

Ishan J. Kelkar et al. [5] this article incorporates a discussion about arranging and gathering an unassuming wind current that can be utilized for instructive purposes. The objective was 
to make satisfactory wind stream at a speed of over $10 \mathrm{~m} / \mathrm{s}$ in the test fragment and to incorporate factors like traditionalism, solace and robustness being developed. Another objective was to direct an investigation on comparable proliferation and show the differentiation among numerical and exploratory outcomes.

Odenir de Almeida et al. [6] this article portrays the subtleties of preparation and building up a medium-sized, subsonic, lowspeed wind stream that is required to show up in the working fragment of $90 \mathrm{~m} / \mathrm{s}$ with a typically low level of unsettling influence and is utilized for research purposes. Ideal speed plan (planes and land vehicles), marking works out, foundational layout applications, vital investigation of liquid mechanics and different potential results.

\section{WORKING PRINCIPLE OF WIND TUNNEL}

Principle of Wind Tunnel

A feature common to all wind tunnels developed according to Bernoulli's theory and the Venturi effect. The Bernoulli principle therefore states that an increase (decrease) in pressure in a circulating liquid must always be accompanied by a decrease (increase) in speed, and vice versa, if an increase (decrease) in the speed of the liquid causes a decrease. (Increase) in pressure. This is at the heart of a number of everyday phenomena. [4]

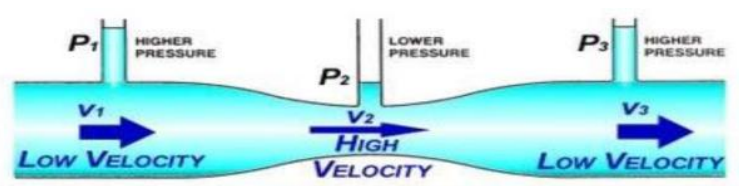

Fig. 1: Principle Bernoulli’s theory

$$
\mathrm{P}+1 / 2 \rho \mathrm{v}^{2}+\rho \mathrm{g} \mathrm{h}=\mathrm{constant}
$$

According to the laws that regulate fluid dynamics, the velocity of a fluid must increase when it passes through a constriction to respect the principle of continuity, while its pressure must decrease to respect the principle of conservation of mechanical energy. Therefore, any gain in kinetic energy that a fluid can accumulate through the restriction due to its increased velocity is cancelled out by a pressure drop.

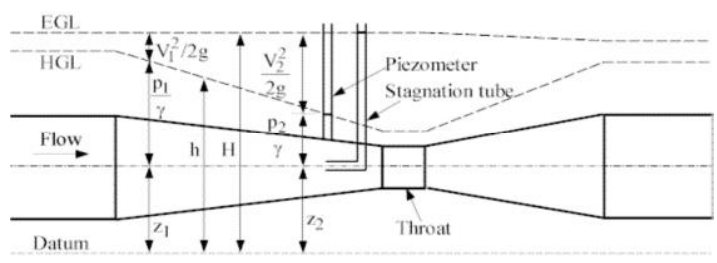

Fig. 2: Principle Venturi effect

\section{Components of Wind Tunnel}

\section{Contraction}

In wind tunnel construction, contraction is used to control flow rate and velocity fluctuations for the test section. By changing the shrinkage ratio (inlet cross-sectional area divided by the outlet cross-sectional area), we can accelerate the wind speed in the test section. To stabilize the flow of wind as it comes out of the contraction, the curve should be smooth on the inside and continue into the next section. The contraction of the blower accelerates and directs the flow into the test section. The size and shape of the contraction determine the intensity levels of the final turbulence in the test section and thus the quality of the flow. In addition, the length of the contraction must be maintained as long as possible to minimize boundary layer growth and reduce the effect of Gortler vortices. The flow out of the contraction should be regular and regular. For a finite length inlet contraction, there is a maximum and a minimum value for the static pressure distribution of the wall along the wall near the inlet and outlet, respectively. Therefore, these two zones can be considered as zones having disadvantageous pressure gradients with a possible separation of the flows. In the event of separation, the smoothness and uniformity of the flow are degraded, which can lead to an increase in the intensity of turbulence in the test section. In summary, it can be said that contractions in wind tunnels generate several unstable lateral currents, which are undesirable and can have considerable effects on the behavior of the downstream boundary layers and the intensity of turbulence in the test section.

Test Section

The test section is the central part of each wind tunnel. The objects are tested and characterized here. Two basic principles follow: the stability of the wind field and the efficiency of the wind tunnel become important. The quality of the airflow in the test section represents the overall performance of a wind tunnel. To achieve high quality wind flow in the test section, several basic criteria must be met:

1) The stability of the wind flow comes first; to achieve this, all designs of the wind tunnel sections must be well integrated and the designer must minimize turbulence.

2 ) To reduce the wind tunnel performance, the cross section should be small enough to increase the wind speed. The shape of the cross section must be carefully selected based on the shape of the object. You can save more space in the construction of cross sections, which saves more money and more electricity. 
3) The blower designer must know the normal speed of the test section in order to optimize the position of the drive fan.

4) The test object and associated equipment must be easy to load.

5) A wind requires a door and window to view or read data.

The cross section should be determined according to the following principles:

1) A good blower should deliver stable and constant speed over approximately $90 \%$ of the test section area. The test pattern cannot be larger than this $90 \%$ area.

2) Taking into account the blocking effect of the model, the surface of the test model should normally not exceed 5\% of the cross section of the wind tunnel.

3) The walls of the wind tunnel can interrupt the flow of wind if the wings of the test model are too long and exceed $60 \%$ of the width of the wind tunnel.

Settling chamber

Screens are essential devices for reducing turbulence in wind tunnels. Screens are used to compensate for the variation in the flow velocity of the sedimentation section. Fine vertebral structures can be removed and honeycombs can remove large vertebral structures. They also break large eddies into small eddies that disintegrate rapidly over short distances. Honeycombs and screens are two useful tools in the settling chamber to stabilize the wind flow and minimize turbulence. After the air has passed the contraction, different wedges and the driving fan, the generated turbulence and the wind speed are not the same in the vector and the velocity figure. This is why we need a settling chamber to compensate for the wind speed and ensure the wind stability of the test section.

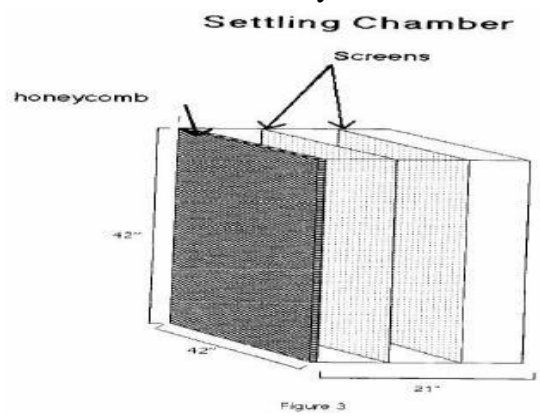

Fig. 3 Honeycomb and screens in settling chamber

Similar to honeycombs, gauze is a very effective tool that can be used to lay chambers to reduce turbulence and irregularities. The more layers, the higher the stabilization efficiency. However, relative resistance also increases. To get accurate data, we only used the honeycomb structure for stabilization in this study, taking into account the power of the drive fan.

Component Design Guidelines
This section describes the design of various tunnel components including flow conditioner, shrinkage, test section, diffuser and fan drive. Optional components such as a rotating section, vibration isolator and jet manifold for open jet test sections are also discussed.

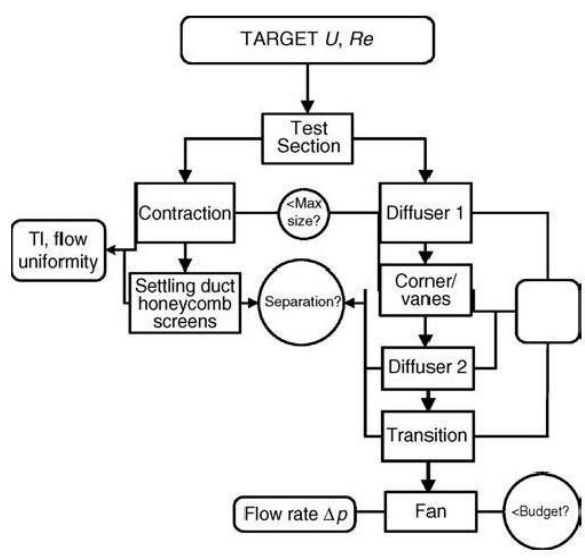

Fig. 4 Flow chart for design of Wind tunnel

Flow conditioner

In most tunnels, the flow conditioning section includes a honeycomb, screens and a sedimentation channel. An example of a honeycomb section is shown in Figure 5. The honeycomb aligns the flow with the axis of the tunnel and dissolves the largest flow fluctuations. Screens go from large turbulent fluctuations to smaller scales. These disintegrate in the sedimentation channel, which must be long enough to allow for sufficient disintegration and at the same time minimize boundary layer growth.

The honeycomb eliminates eddies from the oncoming river and minimizes lateral fluctuations in mean velocity and oscillation velocity (Mehta and Bradshaw, 1979). The yaw angle of the oncoming river should be less than $10^{\circ}$ to avoid stalling of the honeycomb cells. Honeycombs come in a variety of shapes such as circular, square and hexagonal cross sections. Of these, the hexagonal shape is generally the crosssectional shape chosen because it has the lowest pressure drop coefficient (Barlow, Rae \& Pope, 1999). Honeycomb cells have been shown to perform best with a length to diameter ratio of 7 to 10 (Mehta and Bradshaw, 1979). Mehta and Bradshaw (1979) also indicate that the cell size should be less than the smallest lateral wavelength of the velocity change. The honeycomb section should have sufficient structural rigidity to withstand the forces exerted during operation without significant deformation. Special attention may be required if the number of machines entering the honeycomb section is high enough to allow for flow restriction. 


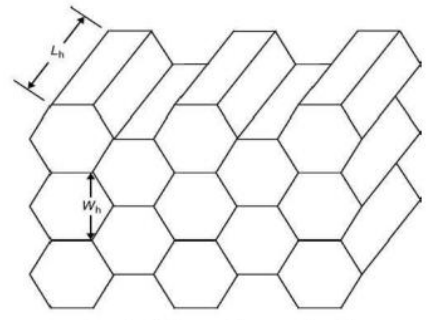

Fig. 5 Construction of Honeycomb

\section{Contraction}

The contraction of the inlet plays a crucial role in determining the quality of the flow in the test section. The contraction accelerates and directs the flow into the test section. The size and shape of the contraction determine the intensity levels of the final turbulence in the test section (Derbunivich et al., 1987). The contraction lengthens the filaments of the vortex, which increases the turbulent but lateral axial fluctuations (Tennekes and Lumley, 1972). The length of the contraction should be small enough to minimize the growth and cost of the boundary layer, but long enough to avoid large unfavorable pressure gradients along the wall that can be created by aerodynamic curvature and lead to flow separation. While CFD can be used in modern design schemes, Morel (1975) proposed a simple method for analyzing the corresponding polynomials. A polynomial diagram of the contraction form is shown in Fig. The input level of the contraction is Hi and the output level is He. The total length of the contraction is $\mathrm{L}$, and the two polynomials are paired in a certain position $\mathrm{x}=\mathrm{xm}$ (position, slope and curvature). At the end of the contraction, all remaining "free" higher order derivatives of the polynomial are set to zero to give a uniform section.

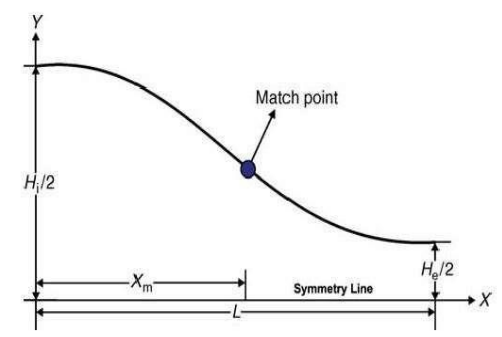

Fig. 6 Schematic of the contraction shape with matched polynomials

\section{Test Section}

The test section can be of the closed wall type or the open beam type. The design of the test section should allow for easy access and installation of the test models and tools in the wind tunnel. The aerodynamic performance of the models may be more suited to full-scale performance in a closed test section. However, an open beam allows far-field acoustic measurements at the expense of possible deflection of the beam from the test section, the interactions between the beam and the collector and the breaking of the shear layer (Amiet, 1978). In a test section with a closed wall, acoustic measurements suffer from poor signal-to-noise ratios due to pressure fluctuations of the turbulent boundary layer [normally] and acoustic reverberation. The main goal of the installation project is to determine the type of test leg that will be used. For 2D studies, the chord should not exceed the range of $13 \sim 23$ (Barlow, Rae and Pope, 1999). It is also important to have a block rate $<10 \%$ based on the frontal area of the model (Barlow, Rae and Pope, 1999). A common rule of thumb when sizing test sections is to have rectangular dimensions with a ratio of approximately 1.4 to 1 .

Diffuser

The diffuser slows the high-speed flow of the test track, allowing the recovery of the static pressure and reducing the load on the propulsion system. The flow field inside the diffuser is influenced by the type of flow exiting the test section. The orientation, size (block) and wake of the wing patterns are some of the factors that influence the diffuser input flow. The diffuser area should gradually increase along its axis to avoid flow separation. As with the contraction sections, the geometry of the diffuser can be optimized. Barlow, Rae and Pope (1999) indicate that for a conical speaker, the half-angle of divergence of the speaker walls must be less than 3.5 for a "good" design. Mehta (1977) states that the diffuser angle for a conical diffuser should be between $5^{\circ}$ (for better flow stability) and $10^{\circ}$ (for better pressure recovery). The flat curves of the Klines speaker (Runstadler, Dolan and Dean, 1975) are generally used for the design (without CFD) of the speakers, as shown in Figure 5. The ratio of AR area between the output and the input of the speaker is plotted with respect to the ratio between the length of the speaker and the height of the speaker inlet. Three regions are displayed on the graph. The design of the diffuser is done by choosing a length for the diffuser, i.e. within the dimensional constraints of the installation.

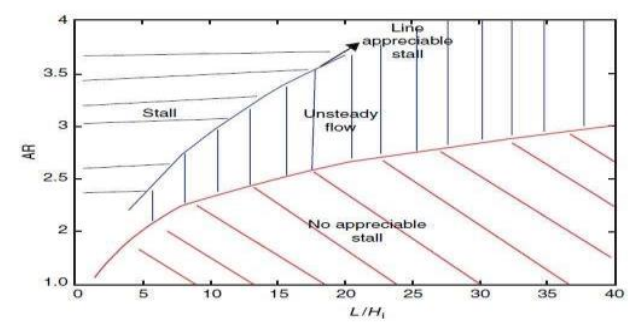

Fig. 7. 2-D diffuser design curves. Adapted from Runstadler, Dolan and Dean (1975)

\section{Drive System Selection}

The drive system generates a volumetric flow rate and compensates for the remaining pressure drops. The driver can be a fan. Pope (1999) provides a method for estimating losses in the circumference of the tunnel, which helps in the selection of fans. Fan performance is characterized by fan load curves, which show diagrams of fan efficiency and pressure drop versus flow rate. The load curves are estimated for different 
fan speeds. The pressure drop calculation (Barlow, Rae and Pope, 1999) leads to the wind tunnel performance curve, which represents an estimate of the static pressure drop for different volume flow values. The intersection points of the pressure drop curves with the tunnel performance curve determine the operating points of the wind tunnel. Fans provide optimum performance when tunnel operating points are close to maximum fan efficiency.

A major component of background noise comes from the fan or drive system. With a ventilator or regulated source of pressurized gas. Fans are classified according to the volumetric flow rate and static pressure drop they can overcome.

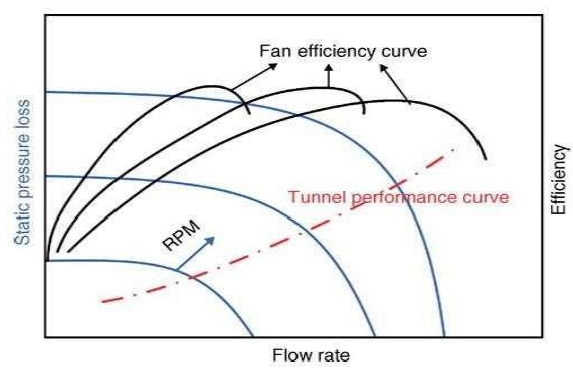

Fig. 8 Fan load curves

When designing a low noise installation, fan noise must be attenuated. The pass frequency of the blades $(\mathrm{BPF}=\mathrm{R} \cdot \mathrm{N}$ blade) and its harmonics appear as discrete tones in the background noise spectra of the test section at a fixed tunnel speed and can contaminate sensor measurements and affect the physics of the flow. A spectrogram (contour diagram of sensor signal strength versus frequency), which is obtained via shortterm discrete Fourier transformations with increasing tunnel speed, is an effective tool for discovering passage contamination. The blade (Duell et al., 2004). This contamination can be reduced by acoustic treatment of the tunnel circuit and other acoustic pathways (to a minimum) between the fan and the test section.

Settling chamber honeycomb
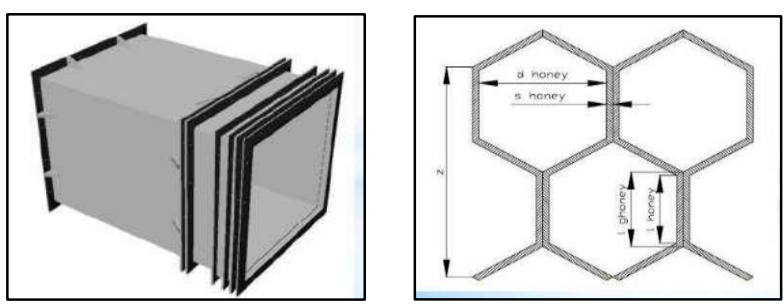

Fig. 9 settling chamber and honeycomb structure

Table no. 1 Dimensions of Wind tunnel.

\begin{tabular}{|l|l|l|l|}
\hline S No. & Components of Wind Tunnel & $\begin{array}{l}\text { Length } \\
(\mathrm{m})\end{array}$ & $\begin{array}{l}\text { Diameter } \\
(\mathrm{m})\end{array}$ \\
\hline
\end{tabular}

\begin{tabular}{|c|c|c|c|}
\hline 1 & Settling Chamber & 0.448 & 0.56 \\
\hline 2 & Converging Section & 0.51 & 0.56 to 0.2 \\
\hline 3 & Test Section & 0.45 & 0.2 \\
\hline 4 & Diverging Section & 1.328 & $\begin{array}{ll}0.2 & \text { to } \\
0.284 & \end{array}$ \\
\hline
\end{tabular}

\section{ANALYSIS Of FLOW}

1 Parameters of the analysis:

Software's used:

- Creo parametric

- Ansys workbench

Material of the configuration:

- $\quad$ Outer body - wood $\square$ Testing object - aluminium

Boundary Conditions:

- Inlet temperature of air : $26^{\circ} \mathrm{C}$

- Inlet velocity : $20 \mathrm{~m} / \mathrm{s}$

- Inlet pressure: 1.01325 bar(atm pressure)

- Outlet condition: Pressure outlet 8.2 Analysis with cylindrical object

1 Creo model:

3D modelling

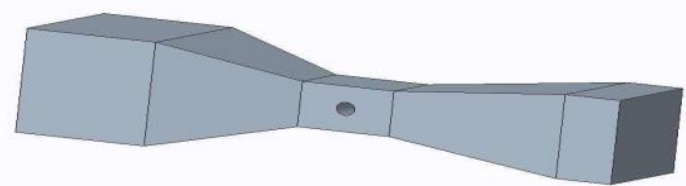

Fig 10 Creo model of wind tunnel

The wind tunnel was modeled with parametric Creo. The object to be tested is a solid cylinder with a diameter of 100 $\mathrm{mm}$. The dimensions of the converging section, the test section and the diverging section are the same as those mentioned above. The only difference is that the settling chamber is not equipped with honeycomb screens for simulation purposes.

Mesh

ANSYS Meshing is a general-purpose, intelligent, automated high-performance product. 


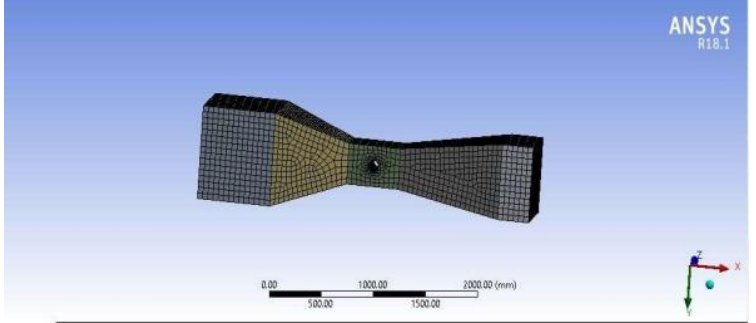

Fig 11 Mesh

Create the most suitable network for precise and efficient multiphase solutions. The main purpose of higher-order elements is to represent the domain boundary more accurately, although they also have advantages of precision within the array. One of the goals of networking is actually to use finite element solutions to make the problem solvable. By networking, you divide the domain into parts, each of which represents an element. Fine mesh is used for greater accuracy. Pressure distribution

The pressure distribution contours of the blower with the cylinder body can be shown as shown in Fig.8.3. As air enters the converging section, its pressure decreases. At the point where the airflow first reaches the test body, we can see a localized increase in pressure. This pressure increase is due to the conversion of velocity into pressure, known as the stagnation phenomenon. The point is known as the stagnation point.

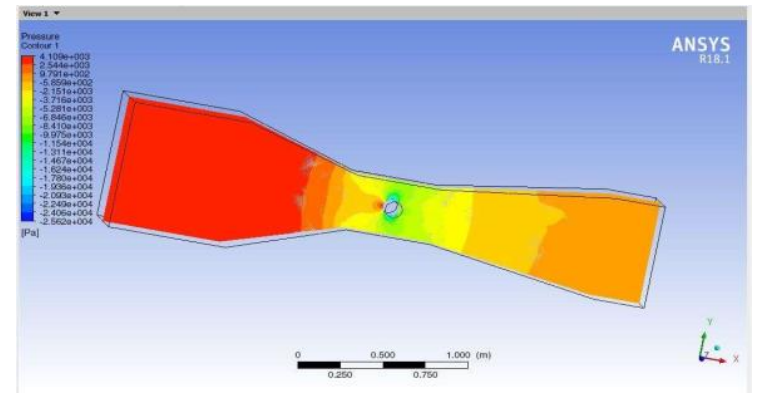

Fig 12 Pressure distribution

Velocity distribution

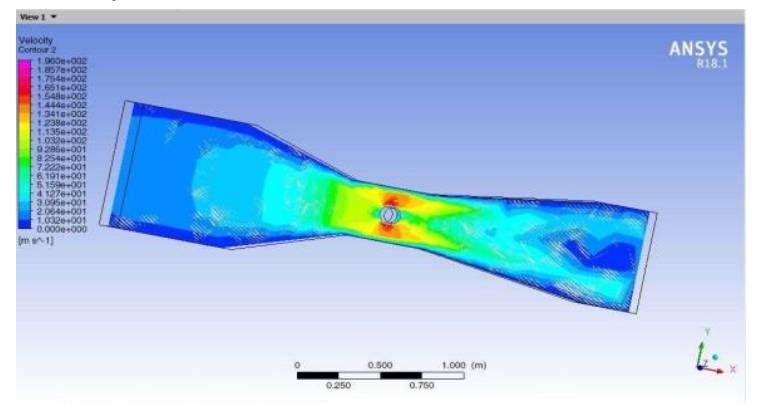

Fig 13 Velocity distribution

The velocity contour of the blower with a cylindrical testing body can be shown as shown in Fig.13. The profile shows that the air speed continues to increase as it passes through the converging section. Again, the speed gradually increases as it passes through the divergent section.
Analysis with aerofoil shape

1 Creo model

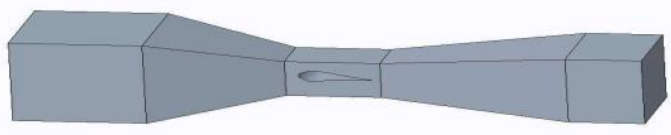

Fig 14 Creo model of wind tunnel with aerofoil shape

3D modelling is done using Creo parameters. A fine mesh is used for the simulation. The iterations considered for the simulation were 100 .

2 Pressure distribution:

The pressure distribution with the wing-shaped test object can be seen in Fig.8.6. The contour is drawn in the central plane ( $\mathrm{z}$ $=0$ ) of the wind tunnel. If we compare the results of the two test objects, we can see that the flow is more rational in the latter case. In this case the air flow is no longer disturbed as before. At the same time, the constitution of the continuity equation and Bernoulli's theorem apply in both cases.

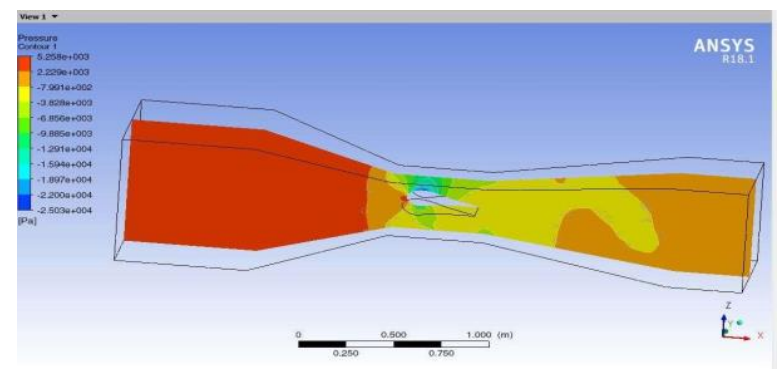

Fig 15 Pressure distribution

3 Velocity Distribution

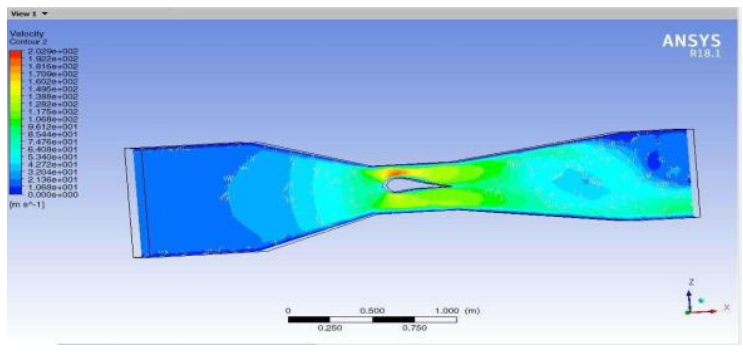

Fig 16 Velocity distribution.

4 Inference from the results:

After examining the velocity profile and pressure profile of the two test objects, we can see that the flow does not converge smoothly and the separation of the flow occurs after the divergent section. They don't meet expectations. The reason may be that the sedimentation chamber is not included in the simulation project, it will be in the actual project. The dimensions used for modeling are derived from empirical relationships that need to be modified based on the simulation results. We can also see that there is a reversal of flow at the end of the divergent section. 


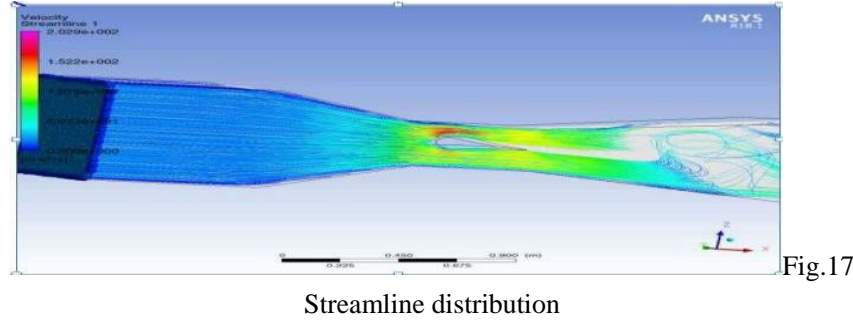

Design Modifications to Remove Flow Reversal

In previous designs, the length of the diverging section was inadequate and therefore the flow reversal occurred in the diverging section. In the next phase of the project, we changed the length of the divergent section and ran the analysis on it. Below are the CAD drawings of the new model:

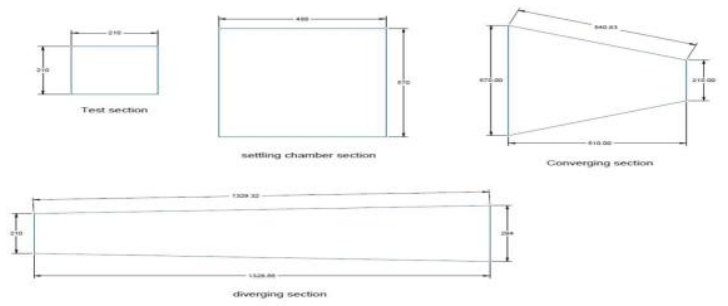

Fig. 18 CAD drawing of sections

Analysis with Cylindrical Object:

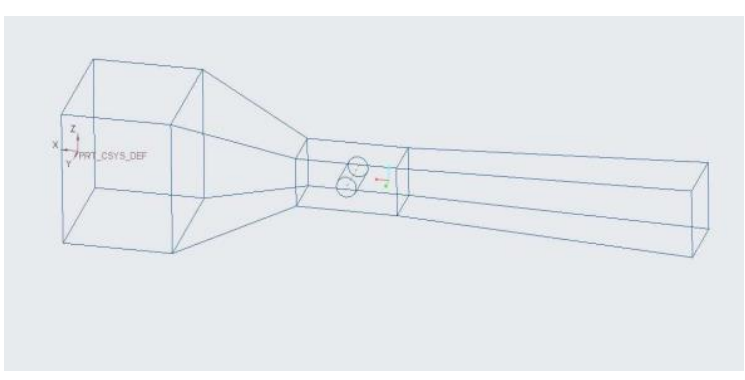

Fig 19 CAD drawing of wind tunnel with cylindrical object 1 Pressure distribution:

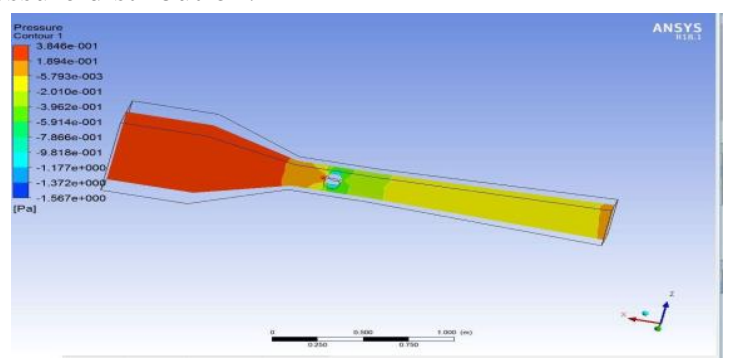

Fig. 20 Pressure distribution of wind tunnel with cylindrical object

2 Velocity distribution:

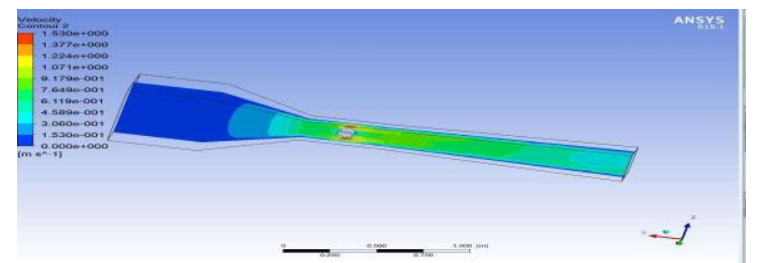

Fig. 21 Velocity distribution of wind tunnel with cylindrical object
3 Streamline distribution:

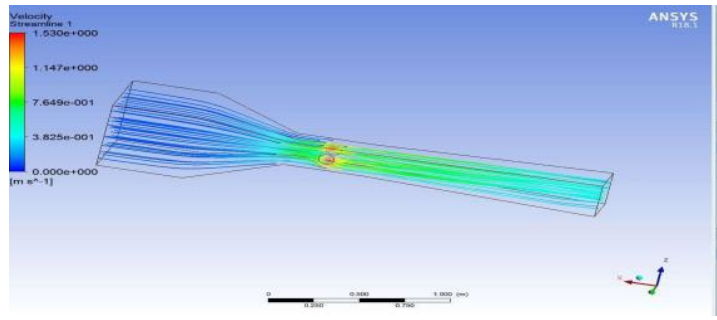

Fig. 22 Streamline distribution of wind tunnel with cylindrical object

4 Flow distribution:

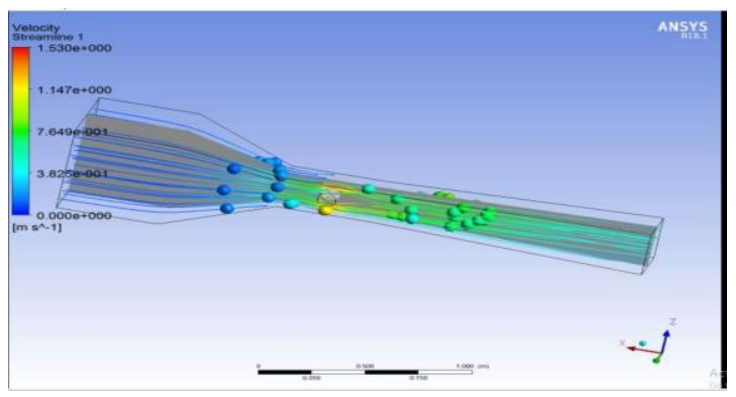

Fig 23 Flow distribution of wind tunnel with cylindrical object Analysis with aerofoil shape:

1 CAD model:

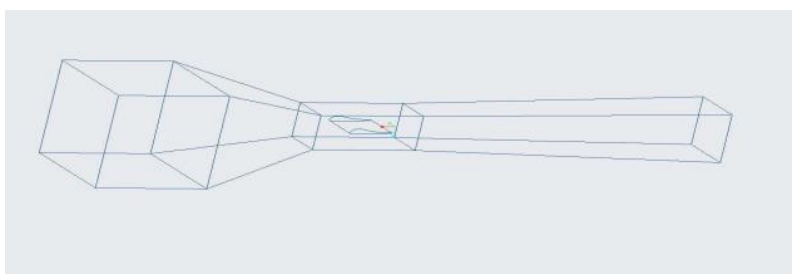

Fig. 24 CAD drawing of wind tunnel with aerofoil object 2 Pressure distribution:

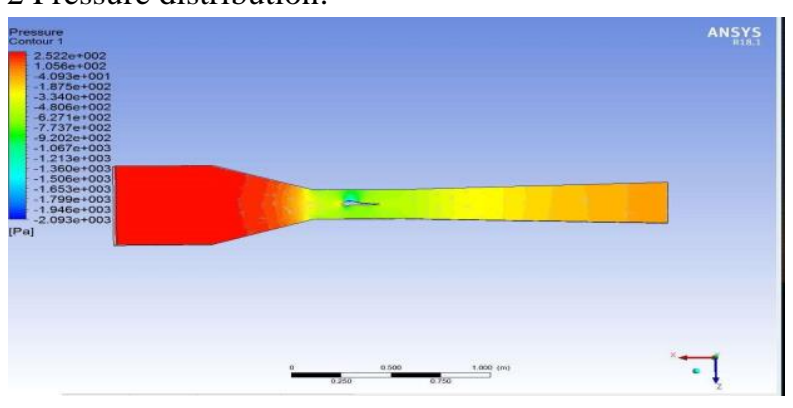

Fig. 25 Pressure distribution of wind tunnel with aerofoil object 3 Velocity distribution: 


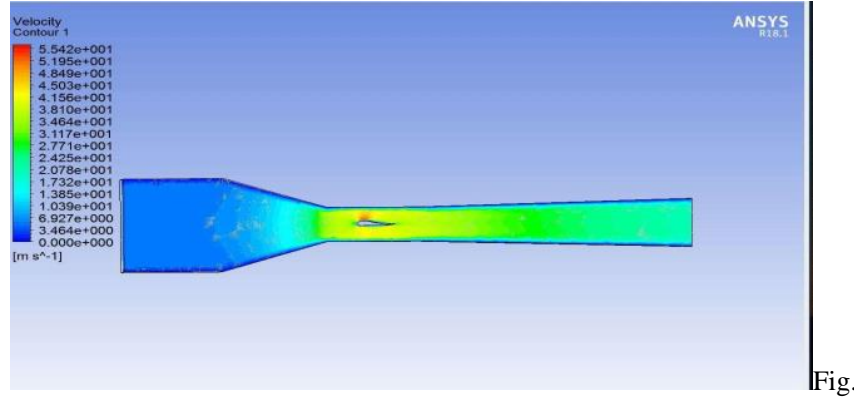

26 Velocity distribution of wind tunnel with aerofoil object 4 Streamlines distribution:

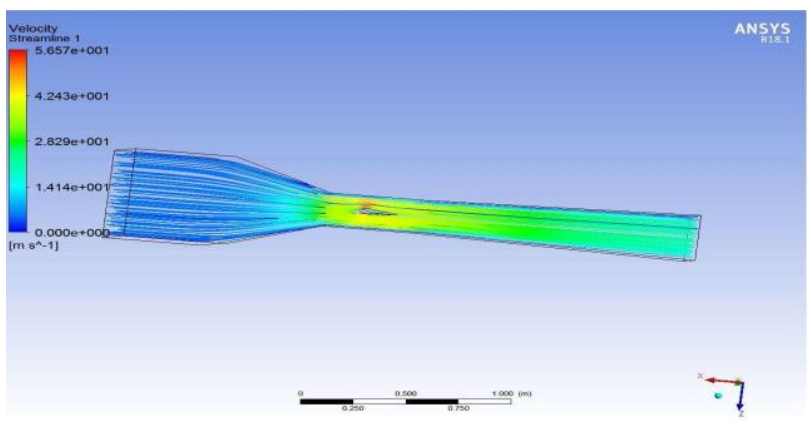

Fig. 27 Streamlines distribution of wind tunnel with aerofoil object

\section{Flow distribution:}

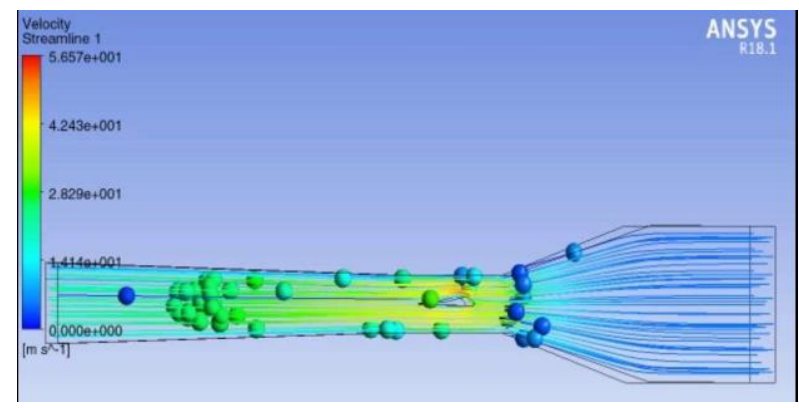

Fig. 28 Flow distribution of wind tunnel with aerofoil object

Material Selection for Wind Tunnel

1 Plywood:

- Material: MDF

- Dimensions: 12 feet*8feet

- Quantity: 2

- Price: 910 Rs/piece $\square$ Advantages:

Good Strong Heavy Board

Very Hard

We can Glue them Paint Them, Screw Them

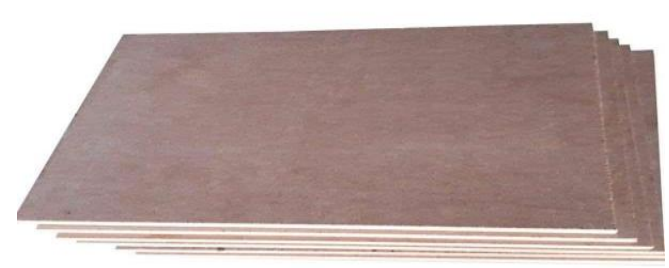

Fig. 29 Plywood

2 Acrylic Sheet

- Material type: cast acrylic

- Size: 12 X 12 inch

- Thickness: $4 \mathrm{~mm}$

- BENEFITS- Acrylic is stronger and lighter than glass, excellent resistance to longterm exposure of outdoor elements

- Price: 399Rs/Piece

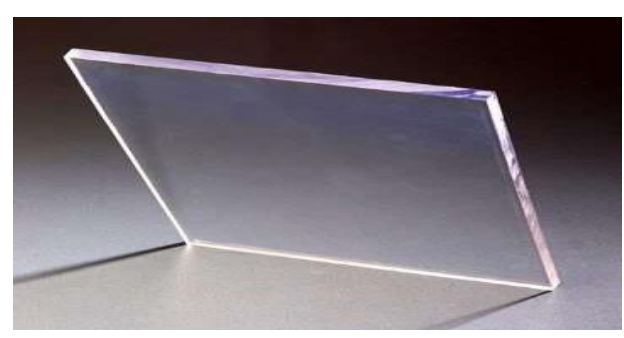

Fig. 30 Acrylic sheet

3 Honeycomb

- Manufacturer: symphony air cooler

- Material: Evaporative Cooling Pad is made of wood-pulp paper with a layer of glue, which is anti-corrosion, antimustiness and has good water absorption

- Price: $945 \mathrm{Rs}$

- Dimensions: 538(H)*223(W)*38(D)

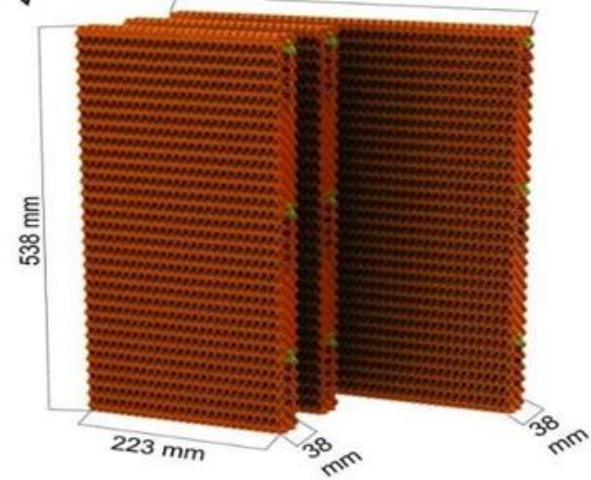

Fig. 31. honeycomb

10.4 Specification of fan

- Manufacturer: Digismart

- Type - Axial Fan 
- Diameter - 12 inch

- Power - $22 \mathrm{~W}$

- Maximum Flow rate $-60000 \mathrm{~m} 3 / \mathrm{hr}$.

- $\quad$ Speed - $1400 \mathrm{rpm}$

- Price- 1990Rs

- Advantage:

\section{Less Noisy}

Easily Inverter Operational Due to Low Power Consumption Feature

It has speed controller

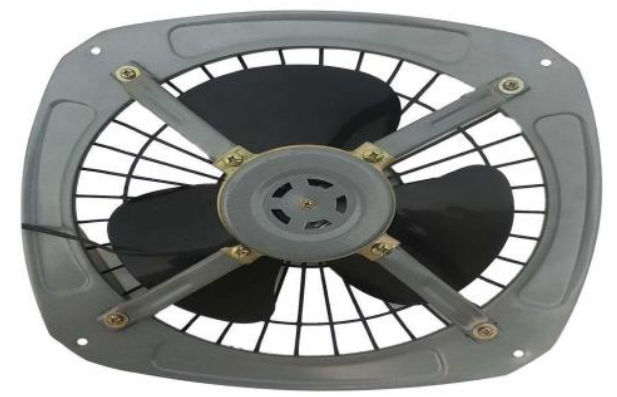

Fig. 32 Fan

Wind Tunnel Omega WT-4401 And WTM-1000

Calibration of Pitot tube using WTM-1000

\section{CONCLUSION}

In this project, the wind tunnel was designed and analysed one by one at low speed. It was concluded that the shape, size, choice of materials and the manufacturing process are very important for the fabrication of a wind tunnel. The results show that the velocity increased and the pressure decreased as the height of the object gradually increased along the length to the maximum and after the maximum height the pressure was increased and the velocity decreased. The wind tunnel was tested on cylindrical objects and wing objects. In the first part of the design, the reversal occurred in the divergent section, but after the correction in the design, the reversal did not occur. To control the flow in the laminar model, the honeycomb structure was inserted into the test section to stop the flow. We performed a calibration comparison of three different flow meters. The main plan was to make the slow wind tunnel model, but due to the Covid-19 pandemic, we were unable to complete production.

\section{REFERENCES}

[1] Sahith Reddy Madara, Jerrin Thadathil Varghese, "Design And Fabrication Of Low Cost Open Circuit Subsonic Wind Tunnel", IJITR, vol. 5 issue 3 april 2017.

[2] Amol L. Mangrulkar, Shubham Bagade "Design and Fabrication of an Open Circuit Subsonic Wind Tunnel for Educational Purposes", International Research Journal of Engineering and Technology (IRJET) e-ISSN: 2395-0056 Volume: 06 Issue: 04 | Apr 2019.

[3] Ishan J. Kelkar, Shreekumar S. Kamalapurkar "Design, Fabrication and Testing of Low-Subsonic Open-Circuit Wind Tunnels - A Review", International Journal of Engineering Research \& Technology (IJERT), Vol. 8 Issue 10, October-2019.

[4] Odenir de Almeida, Journal of Aerospace Technology and Management Print version ISSN 1984-96480n-line version ISSN 2175-9146 J. Aerosp. Technol. Manag. vol.10 São José dos Campos 2018 Epub Feb 26, 2018.

[5] Rae, W. H. Jr. and Pope A.,Low-Speed Wind Tunnel Testing, John Wiley \& Sons, 2nd Ed., 1984.

[6] Tsien, H. S., "On the Design of the Contraction Cone for a Wind Tunnel”, J.Aeronautical Science, pp. 68-70, 1943.

[7] Fundamentals of Wind-Tunnel Design, Louis Cattafesta, Chris Bahr, and Jose Mathew, department of Mechanical and Aerospace Engineering, University of Florida, Gainesville, FL, USA

[8] Low Subsonic Wind Tunnel - Design and Construction, Odenir de Almeida, Frederico Carnevalli de Mirande, Olivio Ferreira Neto, Fernanda Guimarães Saad.

[9] Laws, E. M. and Livesey, J. L., "Flow through Screens", Annual Review of Fluid Mechnics, Vol. 10, pp.247-266, 1978.

[10] Wind tunnel techniques by Sangram Samal

[11] Manual book for OMEGA WT-4401

[12] Manual book for OMEGA WTM-1000

[13] Schubauer, G. B., Spangenberg, W. G., Klebanoff, P. S., "Aerodynamic Characteristics of Damping Screens", NACA TN 2001, 1950. 